\title{
Nogo-B receptor in relevant carcinoma: Current achievements, challenges and aims (Review)
}

\author{
YU-KUN LI ${ }^{1 *}$, YUAN-JIE XIE ${ }^{1 *}$, DAI-CHAO WU ${ }^{1}$, SHUANG-LIAN LONG ${ }^{1}$, \\ SHENGSONG TANG ${ }^{2}$ and ZHONG-CHENG MO ${ }^{1}$ \\ ${ }^{1}$ Department of Histology and Embryology, Clinical Anatomy and \\ Reproductive Medicine Application Institute, University of South China, \\ Hengyang, Hunan $421001 ;{ }^{2}$ Department of Pharmacology, \\ Hunan University of Medicine, Huaihua, Hunan 418000, P.R. China
}

Received March 15, 2018; Accepted July 17, 2018

DOI: 10.3892/ijo.2018.4520

\begin{abstract}
The novel neurite outgrowth inhibitor B (Nogo-B) receptor $(\mathrm{NgBR})$ is specific for Nogo-B, which is highly expressed in various human organs and cells, including the lung, liver, kidney, smooth muscle cells, blood vessel endothelial cells and inflammatory cells. Previous studies have indicated that $\mathrm{NgBR}$ directly interacts with Nogo-B and is able to independently influence lipid and cholesterol homeostasis, angiogenesis, N-glycosylation, the epithelialmesenchymal transition, the chemotaxis of endothelial cells and cellular proliferation and apoptosis. These multiple functions and actions of this receptor provide an understanding of the important roles of $\mathrm{NgBR}$ in various conditions, including fatty liver, atherosclerosis, intracranial microaneurysms, retinitis pigmentosa and severe neurological impairment. Furthermore, NgBR has been demonstrated to exert protean, multifunctional and enigmatic effects in cancer. The present review summarizes the latest knowledge on the suppressing and activating effects of $\mathrm{NgBR}$, emphasizing its function in cancer. Further basic and medical research on this receptor may provide novel insight into its clinical implications on the prognosis of relevant human cancer types.
\end{abstract}

Correspondence to: Dr Zhong-Cheng Mo, Department of Histology and Embryology, Clinical Anatomy and Reproductive Medicine Application Institute, University of South China, 28 West Changsheng Road, Hengyang, Hunan 421001, P.R. China

E-mail: zhchmo@hotmail.com

Dr Shengsong Tang, Department of Pharmacology, Hunan University of Medicine, 492 South Jinxi Road, Huaihua, Hunan 418000, P.R. China

E-mail: tangss111@163.com

*Contributed equally

Key words: neurite outgrowth inhibitor B receptor receptor, invasive ductal breast carcinoma, hepatocellular carcinoma, non-small cell lung carcinoma, multiple myeloma

\section{Contents}

1. Introduction

2. Expression of $\mathrm{NgBR}$ in cancer

3. Potential mechanism of $\mathrm{NgBR}$ in malignant tumour types

4. Conclusions and future perspectives

\section{Introduction}

Neurite outgrowth inhibitor B (Nogo-B) receptor (NgBR), a type I transmembrane protein with multiple functions and actions at the plasma membrane that is mainly expressed in the lung, breast, smooth muscle and blood vessels and is widely distributed in the liver, is able to interact with Nogo-B and vascular endothelial growth factor (VEGF) or function independently to exert multiple effects (1-4). This protein is a key member of the reticulon (RTN) protein family. The reticulon isoforms of mammalian RTN1, RTN2, RTN3 and RTN4c proteins have been identified, and these form the largest branch of the reticulon superfamily (4-6). NgBR contains a putative residue signalling sequence in the $\mathrm{N}$ terminus, an extracellular domain, a single transmembrane region and a cytoplasmic domain, which are composed of residues 1-46, 47-119, 120-139 and 140-293, respectively (7). As indicated in the database of the National Centre for Biotechnology Information, the gene encoding $\mathrm{NgBR}$ in humans, Nus1, dehydrodolichyl diphosphate synthase subunit, may be located at 6q22.1 (8). Previous studies have provided an increasing body of evidence suggesting that $\mathrm{NgBR}$ has different cellular localizations and controls specific membrane transport pathways in numerous human diseases, including fatty liver, intrauterine pulmonary hypertension, atherosclerosis, protozoan infections, retinitis pigmentosa, congenital disorders of glycosylation and relevant cancer types $(3,9)$.

A previous circular dichromatogram analysis indicated that the full-length $\mathrm{NgBR}$ macromolecule has a high content of helical structures, and its structure comprises $\sim 38 \% \alpha$-helices, $15 \% \beta$-sheets and $47 \%$ random coils in the ectodomain and the cytoplasmic domains (5). The ectodomain of NgBR, a member of the intrinsically unstructured protein family, is 
characterized as intrinsically unstructured without secondary and tertiary structures (5). The NgBR cytoplasmic domain has partially secondary structures without tight tertiary packing and serves as a scaffold that utilizes short motifs for the assembly of two binding partners, which are isoprenyl lipids and/or prenylated proteins $(5,7)$.

Activation of the Nogo- $\mathrm{B} / \mathrm{NgBR}$ signalling axis may modulate angiogenesis (10), lipid and cholesterol metabolism (11) and $\mathrm{N}$-linked glycosylation (12) and enhance axonal branching (13) and cellular proliferation and apoptosis (10) under different physiological and pathological conditions by interacting with multiple downstream targets, including mammalian target of Niemann-Pick type C2 (11), cisprenyltransferase (12) and endothelial nitric oxide synthase (eNOS (10). More importantly, targeting this signalling axis has been revealed to be promising for the treatment of cancer. It has been predominantly reported to have an oncogenic function and elevated expression in certain cancer types, including invasive ductal breast carcinoma (IDC) (14), hepatocellular carcinoma (HCC) (15), malignant melanoma (MM) (16) and non-small cell lung carcinomas (NSCLC) (17), which implies that targeting this signalling axis may have potential for retarding or ameliorating cancer progression.

In summary, $\mathrm{NgBR}$ serves a notable role in a number of human diseases, particularly cancer, which is a highly studied and complex subject (3). Once the first report of its effect on breast cancer was published in 2013 (14), numerous studies on the function of $\mathrm{NgBR}$ in the mechanism of cancer have been reported, and NgBR has gradually become a novel research hotspot globally (14-17). However, no systematic review of the latest research results for $\mathrm{NgBR}$ in the cancer research field has been published, to the best of our knowledge. The present review assesses and discusses novel advances achieved in previous studies on $\mathrm{NgBR}$, with a particular emphasis on the expression and pathophysiological effects of $\mathrm{NgBR}$ on relevant human cancer types (17), including IDC (14), MM (16), HCC (15) and NSCLC (17). The present review additionally assessed the data implicating $\mathrm{NgBR}$ in the regulation of lipid metabolism (11), N-linked glycosylation (12) and the epithelial-mesenchymal transition (EMT) (18). These results identify $\mathrm{NgBR}$ as a novel and potential therapeutic target for mitigating the complex disease states in relevant human cancer types.

\section{Expression of $\mathrm{NgBR}$ in cancer}

Researchers have demonstrated that $\mathrm{NgBR}$ is recognized as a highly important receptor associated with a number of cancer types, including IDC (14), HCC (15), MM (16) and NSCLC (17). Furthermore, longitudinal studies have revealed that the dysregulation of $\mathrm{NgBR}$ over time is a risk factor for the early development of these cancer types (14-17). NgBR is able to promote the development of IDC (14) and HCC (15) but has an inhibitory effect on MM (16) and NSCLC (17). Therefore, there is some dispute regarding the expression and mechanism of $\mathrm{NgBR}$ in different types of malignancies.

Upregulated expression of $\mathrm{NgBR}$ in relevant cancer types. Levels of $\mathrm{NgBR}$ are increased in IDC. IDC, a severe public health problem globally, represents a frequent cause of cancer-associated morbidity and primarily occurs in women aged 20 to 59 years, resulting in a huge socioeconomic burden (19).

The contribution of NgBR-derived factors to cancerinduced mammary gland disease is an emerging area of research. A study conducted by Wang et al (14) revealed that the NgBR levels are higher in IDC compared with in normal breast tissue and are strongly associated with estrogen receptor $(\mathrm{ER})(+)$ and human epidermal growth factor receptor 2 (HER2)(-) breast carcinoma. Furthermore, these observations suggest a strong association between altered $\mathrm{NgBR}$ protein expression and survivin gene expression in IDC. Oestradiol is able to increase survivin expression and proliferation (14). Interestingly, the downregulation of $\mathrm{NgBR}$ is able to decrease the effect of oestradiol on survivin expression and cellular proliferation (14). An inverse correlation has been revealed between NgBR expression and the expression of Ki-67 antigen, which is a negative prognostic factor for the initiation, promotion and progression of breast carcinoma (20). Furthermore, the malignancy grade of IDC has a close association with the expression of $\mathrm{NgBR}$ but no notable association with the levels of HER2, ER or progesterone receptor, the size of the primary tumour or lymph node metastasis (20). Different associations amongst $\mathrm{NgBR}, \mathrm{ER}(+/-)$ and HER2(+/-) were revealed by Pula et al (20) and Wang et al (14), and the contradictory results in $\mathrm{NgBR}$ expression are likely due to differences in the socioeconomic status, race/ethnicity, geography, age and sex of the patients whose breast tumour tissues were tested. Furthermore, the studies used different antibodies produced by Imgenex (Novus Biologicals, LLC, Littleton, CO, USA) (20) and Epitomics (Abcam, Cambridge, UK) (14), which may have resulted in opposing conclusions. Further clinical discoveries through cell biology experiments are required. The study conducted by Wang et al (14) suggested that tissue sections from human patients with early onset IDC indicate a pattern of $\mathrm{NgBR}$ overexpression.

Levels of $\mathrm{NgBR}$ are increased in HCC. $\mathrm{HCC}$ is the fifth most common carcinoma in humans and the second most common cause of cancer-associated mortality globally (21). The expression of NgBR in HCC tissues is notably higher compared with that in their corresponding adjacent liver tissues. Western blot analysis was used to examine the levels of tumour protein p53 and $\mathrm{p} 21$ proteins in Bel7402 and chemoresistant HCC cells (Bel/5FU) (15). The results indicated that the protein levels of p53 and downstream p21 are downregulated more in Bel/5FU compared with in Bel7402 cells. In addition, these researchers also examined the effects of $\mathrm{NgBR}$ knockdown on p53 protein expression (15). A previous study revealed a notable association between p53 ubiquitination and 5-FU chemoresistance in patients with HCC and chemoresistant HCC cells (Bel/5FU) $(15,22)$. However, clinical pathological parameters including sex, hepatitis history, liver cirrhosis, maximal tumour size, tumour number, vascular invasion and Tumour Node Metastasis stage are not specifically associated with $\mathrm{NgBR}$ expression (15). However, clinical data analyses indicated that patients with high NgBR levels have notably lower survival rates compared with those with low $\mathrm{NgBR}$ levels (15).

Downregulated expression of NgBR in relevant cancer types. Levels of $\mathrm{NgBR}$ are decreased in MM. MM is a severe public 
health problem globally. The spread of MM cells from a MM primary tumour to form metastases at distant sites is the most life-threatening complication and the main cause of mortality in patients with MM (23). Metastasis is a complex, multistep and multifactorial process involving interrelated interactions between tumour and normal cells. Single MM cells translocate to distant secondary sites, thus these cells must dissociate and migrate away from the primary tumour (24).

Due to the consistent neuroectodermal origin of MM and the involvement of the RTN protein family, $\mathrm{NgBR}$ may serve an essential role in the development and progression of MM. Current research on MM metastasis aims to develop a greater understanding of the interactions between the depth of invasion of primary MM tumour cells and the $\mathrm{NgBR}$ levels. A study conducted by Calik et al (16) indicated that $\mathrm{NgBR}$ may serve an important role in reducing the migration and invasiveness of melanoma cells. Conversely, it has been suggested that $\mathrm{NgBR}$ serves an oncosuppressive role in MM. Future investigations with larger patient cohorts and in vitro studies may help to characterize the function of $\mathrm{NgBR}$ in $\mathrm{MM}$ biology (16).

Levels of NgBR are decreased in NSCLC. The most common respiratory cancer is lung carcinoma, which has a prevalence of $\sim 12 \%$ among patients with newly diagnosed cancer. Furthermore, an increased incidence rate has been observed (19). Current therapy for NSCLC aims to relieve pain and control symptoms rather than interfere with the pathophysiology $(17,25)$. A decrease in the levels of $\mathrm{NgBR}$ and Nogo-B have been observed in various cells, including pneumocytes, bronchial epithelial cells of non-malignant lung tissue (NMLT) and NSCLC cancer cells (17). In patients with NSCLC, low NgBR mRNA expression levels result in progressive and generalized increases in the primary tumour size, lymph node involvement and advanced disease stages (15), resulting in exaggerated mortality rates due to an unfavourable prognosis (17). This indicates that NgBR may potentially serve a role in tumour growth and lymph node involvement in patients with NSCLC and NMLT; however, this hypothesis requires further investigation (17). However, a study by $\mathrm{Wu}$ et al (25) revealed that the levels of NgBR in NSCLC tissues were higher compared with those in corresponding tumour cell-positive lymph nodes.

The lung cancer tissues used in the study conducted by $\mathrm{Wu}$ et al (25) were obtained from patients with different socioeconomic statuses, races/ethnicities, geographies, ages and sexes, which may have resulted in contradictory results regarding $\mathrm{NgBR}$ expression. Furthermore, the antibodies used in these previous studies were purchased from Abcam (25) and Imgenex (Novus Biologicals, LLC) (17), respectively, and the use of these different antibodies may result in opposing conclusions. Therefore, these clinical discoveries obtained from cell biology experiments require further validation.

\section{Potential mechanism of $\mathrm{NgBR}$ in malignant tumour types}

Dysregulation of the NgBR mechanism is a well-recognized aspect of cancer biology, and numerous therapeutic strategies rely on targeting cancer by altering cellular metabolic pathways (15). Until now, the pathophysiology of $\mathrm{NgBR}$ in cancer has remained elusive, and research has mainly focused on its potential mechanisms in malignant tumour types (3).

\section{Carcinogenic mechanism of $\mathrm{NgBR}$}

$\mathrm{NgBR}$ facilitates the phosphatidylinositol-3-kinase/protein kinase $B$ (PI3K/Akt) pathway. The PI3K/Akt pathway has been implicated in the carcinogenesis of certain tumour types, and the effects are attributed to the regulation of cell growth, survival, metabolism and apoptosis in physiological conditions and are involved in the development and survival of multiple solid tumour types, including IDC (26).

Wang et al (14) studied the pathogenesis of breast cancer, and the results suggested that the expression of $\mathrm{NgBR}$ is essential for oestradiol-stimulated IDC cell growth and survivin expression. Furthermore, the upregulation of $\mathrm{NgBR}$ is associated with $\mathrm{ER}(+) / \mathrm{HER} 2(-)$ breast carcinoma (14). Zhao et al (27) revealed that the inhibition of $\mathrm{NgBR}$ is able to attenuate the activation of the Akt signalling pathway and subsequently decrease cellular growth, migration, survival and proliferation. A follow-up study revealed that the overexpression of $\mathrm{NgBR}$ enhanced the phosphorylation of Akt in MDA-MB-231 cells (28). Pula et al (20) revealed that NgBR is substantially increased in IDC at the protein and mRNA levels and concluded that a low expression of $\mathrm{NgBR}$ in IDC is able to downregulate the $\mathrm{PI} 3 \mathrm{~K} / \mathrm{Akt} / \mathrm{mech}$ anistic target of rapamycin (mTOR) signalling pathway and subsequently reduce cancer cell proliferation, migration, adhesion, survival and invasiveness. These research results clearly demonstrate that $\mathrm{NgBR}$ may mediate the PI3K/Akt/mTOR signalling pathway and thereby serves a major role in the pathogenesis of IDC.

Mouse double minute 2 homolog (MDM2) is a bona fide ubiquitin ligase for p53 that results in altered transcription in p53 disorders, indicating that it serves a role in the stability of p53 (29). p53, a nuclear phosphorylated protein, inhibits the transformation of human cancer cells (30). One previous study has suggested that the phosphorylation and nuclear translocation of MDM2 and the ubiquitination and destruction of p53 are promoted by the phosphorylation of Akt (31). A gradual and orderly change in the PI3K/Akt/MDM2 pathway caused by $\mathrm{NgBR}$ activation is considered an important mechanism in the pathogenesis of HCC (15). Researchers have observed that increased levels of $\mathrm{NgBR}$ enhance the phosphorylation of Akt, and that phosphorylated Akt subsequently upregulates the expression of phosphorylated MDM2, and phosphorylated MDM2 in turn mediates p53 ubiquitination (15). These changes in the signalling pathway will mediate the emergence of chemoresistance in HCC and may induce cancer development and progression (15). These data clearly demonstrate that the high levels of $\mathrm{NgBR}$ in patients with $\mathrm{HCC}$ bind to phosphorylated Akt and recruit MDM2 to the nucleus, which is an essential step for the activation of PI3K/Akt/MDM2 signalling and tumourigenesis in HCC cells.

NgBR upregulates the EMT. EMT, a well-recognized physiological phenomenon in embryonic development that promotes the migration of neural crest cells out of the neuroectoderm (32), is a critical contributor to the progression of breast cancer as it not only reinforces that epithelial cells lose their differentiated characteristics but also enhances 
their migratory and invasive abilities by the acquisition of mesenchymal features, as indicated by the overexpression of vimentin, a typical marker of mesenchymal cells (33). Importantly, the expression of epithelial and mesenchymal genes may be regulated by tumour growth factor- $\beta$ (TGF- $\beta$ ) activation, which has been proven to be an upstream regulator of EMT (33). Furthermore, the Akt signalling pathway is able to facilitate all these signalling-mediated EMTs (34).

The breast cancer pathogenesis study conducted by Zhao et al (18) revealed that the TGF- $\beta$-induced expression of zinc finger E-box binding homeobox 1 and twist family bHLH transcription factor may be attenuated by inhibiting $\mathrm{NgBR}$, which is necessary for the progression of EMT. This previous study additionally revealed that the inhibition of NgBR may attenuate the phosphorylation of Akt mediated by TGF- $\beta$. Furthermore, previous studies have suggested that the inhibition of Akt may block the TGF- $\beta$-mediated progression of EMT $(18,34)$. It has been suggested that $\mathrm{NgBR}$ functions as a switch between the mesenchymal-to-epithelial transition and the EMT $(18,35,36)$. In addition, overexpression of the Akt signalling pathway promotes the EMT, which has been well-described in the carcinogenesis of IDC (37). The study of lung cancer pathogenesis conducted by Wu et al (25) revealed that the overexpression of $\mathrm{NgBR}$ requires the activation and plasma membrane localization of Ras to increase the levels of Snail family transcriptional repressor 1 through activation of the mitogen-activated protein kinase kinase (MEK)/extracellular signal-regulated kinase (ERK) pathway, which may downregulate the levels of E-cadherin to promote the EMT in NSCLC cells.

$N g B R$ may promote the epidermal growth factor (EGF)/Ras/ ERK signalling pathway. The EGF/Ras/Raf/ERK signalling pathway is a MEK pathway that may be widely activated $(38,39)$. This signalling pathway is involved in the dysregulation of cell differentiation, proliferation and apoptosis, and tumorigenesis likely involves multiple mutations in this pathway (40). Dysregulation of this signalling pathway may be overactivated by the abnormal expression of numerous cell factors, including $\mathrm{NgBR}$, the mutational activation of EGF-like ligands, and the mutation and overexpression of Ras (28).

In the plasma membrane, the combination of guanosine triphosphate (GTP)-bound H-Ras and K-Ras is reinforced by galectin, which is a cytosolic protein with hydrophobic farnesyl-binding pockets (41). Caveolin-1 has a membranedocking site in its lipid raft domains that binds to guanosine diphosphate (GDP)-loaded H-Ras (42). The hydrophobic cytoplasmic domain of $\mathrm{NgBR}$ has a docking site for binding to prenylated Ras, and this binding promotes the translocation of GDP/GTP-bound H-Ras and K-Ras to the plasmalemma, a step that has a pivotal role in oncogenic signalling by these GTPases (28).

A recent cancer pathogenesis study revealed that $\mathrm{NgBR}$ preferentially binds to farnesylated H-Ras, and its carboxyl terminus composed of hydrophobic residues is essential for integrating with farnesylated Ras to form a steady complex that mediates H-Ras signalling $(28,43)$. Through a cell surface biotinylation assay, Zhao et al (28) revealed that the upregulation of NgBR enhances the amounts of membrane-associated $\mathrm{K}-\mathrm{R}$ as and H-Ras without changing their total levels. It has been reported that $\mathrm{NgBR}$ only mediates the accumulation of Ras at the plasma membrane and does not enhance the levels of Ras (43). In addition, the downregulation of NgBR decreases the EGF-stimulated phosphorylation of ERK and Akt (43). These results are sufficient to suggest that the overexpression of $\mathrm{NgBR}$ is able to enhance the EGF-mediated activation of Ras and its downstream kinases, including Akt and ERK (28). That NgBR is essential for the accumulation of Ras in the tumour cell membrane indicates that $\mathrm{NgBR}$ serves a pivotal role in the oncogenic function of Ras in tumour growth. Furthermore, NgBR may influence the EGF-mediated activation of K-Ras and H-Ras, as K-Ras and H-Ras must be translocated to the cell membrane in order to be activated (44) and the upregulation of K-Ras and H-Ras is thought to mediate downstream kinases throughout the cell (45).

$N g B R$ facilitates angiogenesis. Angiogenesis, an important step in cancer progression, involves the development of novel blood vessels necessary for tumour growth (46). Furthermore, a recent study of cancer cell invasion indicated that angiogenesis serves a key role in the proliferation and motility of cancer cells (47). In the early phase of the carcinogenic process, proliferating cancer cells mediate a proangiogenic microenvironment that increases the amount of supplied nutrients, energy and oxygen (48). VEGF, the primary angiogenic factor produced and secreted by cancer cells, is strongly involved in this progression (49). The VEGF/VEGF receptor (VEGFR) proangiogenic pathway, which is upregulated in human carcinoma, is the most extensively studied pathway (49). eNOS mediated by VEGFR has a notable function in carcinogenesis, including the inhibition of DNA repair systems, the epigenetic regulation of different oncogenic pathways and the inhibition of apoptosis (50). Furthermore, a recent study by Ricciuti et al (51) suggested that angiogenesis promotes the procession of cancer and is responsible for the invasion and metastasis induced by VEGF, eNOS, Akt and ERK.

$\mathrm{NgBR}$ is able to attenuate defects in angiogenesis by promoting eNOS and Akt phosphorylation. Furthermore, $\mathrm{NgBR}$ may facilitate tube formation to ameliorate angiogenesis disorders (52). NgBR is necessary for the development of cerebral blood vessels. Nogo-B and VEGF may interact with $\mathrm{NgBR}$, resulting in the phosphorylation of Akt to reinforce angiogenesis (2). Interestingly, miR-26a may directly interact with NgBR to inhibit the VEGF-NgBR pathway and attenuate the phosphorylation of eNOS, which is able to substantially decrease tube formation, angiogenesis and cellular migration and proliferation (53). These results implicate $\mathrm{NgBR}$ as necessary for angiogenesis and the maintenance of migration.

The targeting of endothelial cell metabolism is an appealing strategy to impact tumour-driven angiogenesis, particuarly given that proangiogenesis proteins, including NgBR, VEGF and eNOS, are thought to mediate endothelial cell migration, proliferation and tube formation; therefore, this NgBR/eNOS/ angiogenesis axis may be a potential therapeutic target in the switch from neoangiogenesis to carcinogenesis (53).

\section{Anticarcinogenic mechanism of $\mathrm{NgBR}$}

$N g B R$ attenuates the Liver $X$ receptor $\alpha(L X R \alpha)$ signalling pathway. Cholesterol, a precursor of bile acids and steroid hormones, is essential for various biological functions, 
including increased tumour angiogenesis, reduced tumour apoptosis and increased tumour cell proliferation. Lipids, the main cell membrane component, serve a pivotal role in cell growth and division during their maintenance of cell integrity (54).

It is thought that lipids are implicated in the development of a number of carcinomas, including breast cancer, lung cancer and HCC. A low level of high density lipoproteincholesterol (HDL-C) was revealed to elevate the risk of breast cancer in 38,823 women in Norway, all between the ages of 17 to 54 years (55). A study on the pathogenesis of breast cancer conducted by Alikhani et al (56) suggested that primary mammary tumour growth and metastasis are substantially accelerated by dyslipidaemia and that cholesterol may upregulate the metastasis and growth of breast cancer. These results indicate that dyslipidaemia serves a key role in the metastasis, recurrence and growth of breast cancer and that hyperlipidaemia is able to increase the risk of breast cancer. In a study of the pathogenesis of liver cancer, Ooi et al (57) revealed that the levels of HDL-C are lower in metastatic liver cancer compared with in primary HCC. A study conducted by Rice et al (58) indicates the involvement of apolipoprotein $\mathrm{E}$ in the induction of lung cancer, which may be due to enhanced cholesterol transport into tumour cells. Altogether, these data suggest that decreasing the levels of lipids and cholesterol may suppress cancer development.

LXR $\alpha$ are primarily expressed in the liver and to a lesser extent in the kidney, small intestine, spleen and adrenal gland (59). A study conducted by $\mathrm{Hu}$ et al (11) suggests that $\mathrm{NgBR}$ integrates the liver $\mathrm{X}$ receptor response element with $\mathrm{LXR} \alpha$, and this interaction serves an important role in lipid synthesis. Furthermore, NgBR prevents the translocation of LXR from the cytoplasm to the nucleus and attenuates the induction of sterol-regulatory element-binding protein (SREBP-1c), the lipid regulatory gene of ATP-binding cassette subfamily A member 1 and fatty acid synthase (FASN), which reduces the lipid levels in vitro. These results suggest that $\mathrm{NgBR}$ is able to mediate $\mathrm{LXR} \alpha$ activation to reduce the levels of lipids.

In summary, these results imply that the NgBR-LXR $\alpha$ axis may reduce the levels of free fatty acid (FFA) and triglyceride (TG) in the liver and plasma to suppress the occurrence and development of cancer types including HCC, breast cancer and lung cancer.

$N g B R$ facilitates the adenosine monophosphate-activated protein kinase $\alpha(A M P K \alpha)$ signalling pathway. AMPK signalling participates in numerous pathophysiologic processes, particularly lipid metabolism and tumourigenesis $(60,61)$ During activation, AMPK $\alpha$ enhances the levels of ATP by regulating the metabolism of fats, proteins and sugars, thus inducing cells to evade death and engage in metastasis and the development of drug resistance $(61,62)$. Furthermore, a previous study suggested that AMPK $\alpha$ is closely associated with the tumour suppressor gene p53 (63). This protein mediates the expression of cellular survival-associated signals, including Akt and mTOR. It has been demonstrated that AMPK $\alpha$ serves an important role in cancer prevention by inducing cell cycle arrest and blocking tumour growth (64). Jones et al (65) previously demonstrated that AMPK $\alpha$ may directly promote the phosphorylation of p53 on serine 15 . Furthermore, the activation of AMPK $\alpha$ may enhance the levels of p53 in addition to its phosphorylation and ultimately induce cell arrest at the G1/S phase (66). Compound C, a cellpermeable pyrazolopyrimidine compound, may function as a reversible and ATP-competitive inhibitor of AMPK (67). In addition, Hadad et al (68) demonstrated that the activation of AMPK $\alpha$ is notably downregulated in biopsies of breast cancer compared with normal tissue, which suggests that AMPK $\alpha$ has a cancer suppressive role. These results suggest that AMPK $\alpha$ has a carcinostatic action in tumourigenesis.

A previous study by $\mathrm{Hu}$ et al (10) revealed that $\mathrm{NgBR}$ may not only enhance the phosphorylation of AMPK $\alpha$ but also upregulate the expression of phosphorylated acetylcoenzyme A carboxylase-1 (ACC-1), which is downstream of phosphorylated AMPK $\alpha$. Ultimately, the activation of ACC-1 and AMPK $\alpha$ represses the expression of FASN, SREBP-1c and stearoyl coenzyme A desaturase- 1 and enhances the levels of FFA and TG. Furthermore, the activation of AMPK $\alpha$ may rescue the translocation of $\mathrm{LXR} \alpha$ mediated by a NgBR deficiency to ensure the maintenance of lipid metabolism $(11,69)$. These results suggest that NgBR is able to mediate AMPK $\alpha$ in order to impact lipid homeostasis and LXR $\alpha$. As mentioned previously, disorders of lipid homeostasis may serve a role in tumourigenesis. The AMPK $\alpha$ axis may reduce the levels of lipids and suppress the translocation of $\operatorname{LXR} \alpha$.

$\mathrm{NgBR}$ is able to promote $\mathrm{N}$-glycosylation to attenuate endoplasmic reticulum stress and the unfolded protein response $(U P R) . \mathrm{NgBR}$ is an extremely conserved subunit of cisprenyltransferase, which is essential for the biosynthesis and phosphorylation of dolichol and ultimately contributes to the maintenance of protein $\mathrm{N}$-glycosylation $(9,70)$. Nevertheless, a NgBR deficiency may induce an $\mathrm{N}$-glycosylation disorder to mediate endoplasmic reticulum stress and thus activation of the UPR (12). These results suggest that NgBR is able to modulate this N-glycosylation/endoplasmic reticulum stress/ UPR signalling pathway.

One previous study suggested that a glycosylation disorder is a common occurrence in human cancer, and that these changes are characteristics of cancer types including breast cancer and NSCLC (71). Furthermore, glycosylation serves a key role in the EMT, cell-cell contact, cancer progression and cancer cell metastasis $(72,73)$. These results indicate that the inhibitory mechanisms of $\mathrm{NgBR}$ on cancer cells may be mediated by the maintenance of $\mathrm{N}$-glycosylation.

Recent studies have further investigated whether the UPR may be involved in intervening in the carcinogenic process and may be a novel therapeutic target for controlling neoplasia development or arresting the progression of the malignant phenotype (77-80). One previous lung cancer study noted that the upregulation of the UPR is able to induce the expression of glucose-regulated protein $78 \mathrm{kDa}$ (GRP78) to suppress apoptosis and resistance to anticancer drugs in lung cancer (74). A previous study indicated that $\mathrm{C} / \mathrm{EBP}$ homologous protein and GRP78 may mediate the development and progression of lung adenocarcinoma (75). Furthermore, the activation of GRP78 is upregulated in lung cancer cells, which are able to induce endoplasmic reticulum stress tolerance against the pharmacological action of anticancer drugs (76). Breast cancer cells may also 


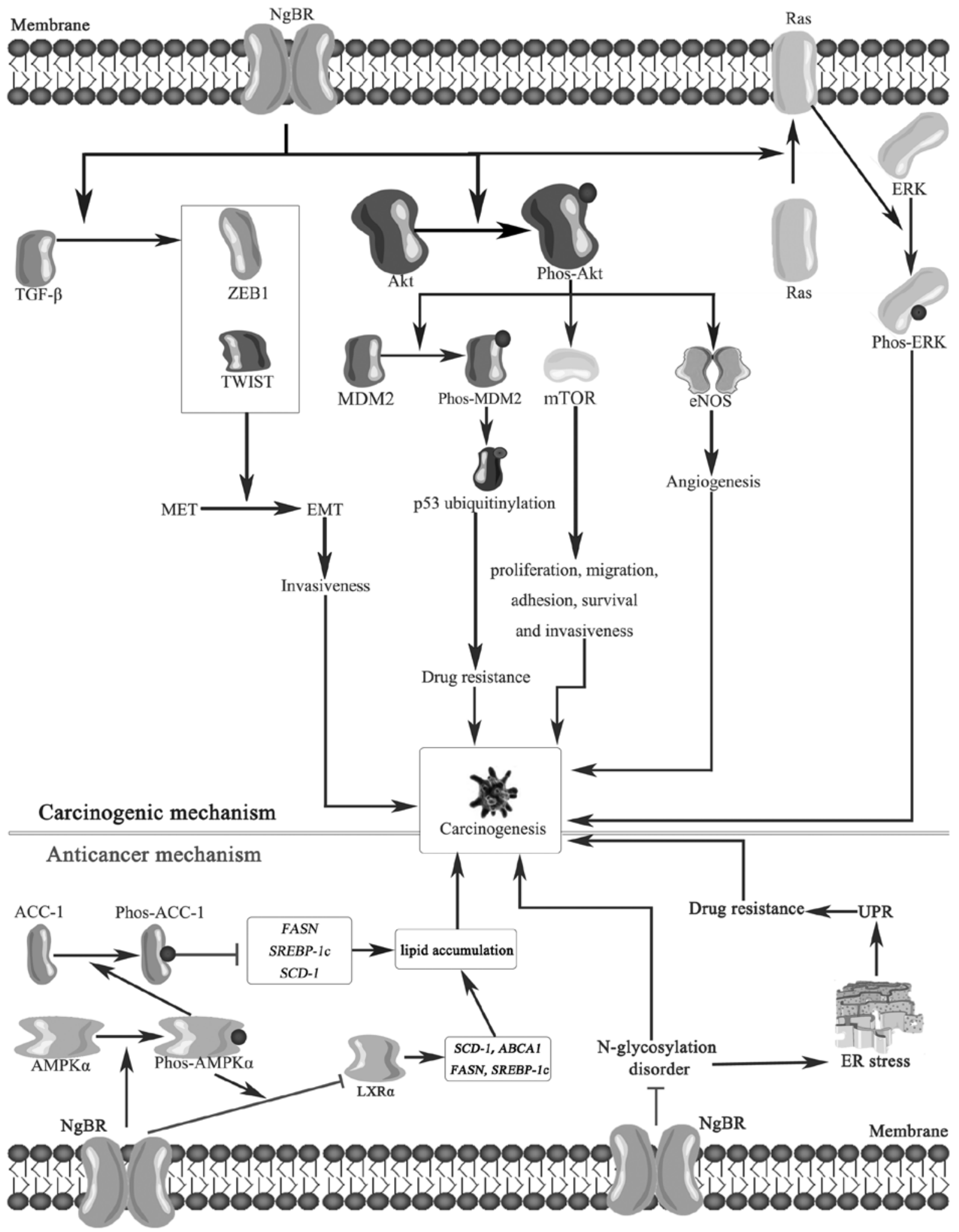

Figure 1. Potential mechanisms underlying the anticancer and carcinogenetic effects of $\mathrm{NgBR}$. $\mathrm{NgBR}$ may promote the switch from the MET to the EMT via TGF- $\beta$, which upregulates the invasiveness of cancer cells. Furthermore, NgBR enhances the phosphorylation of Akt, and phos-Akt subsequently activates mTOR, which promotes cancer cell proliferation, migration, adhesion, survival and invasiveness. Furthermore, activated Akt enhances the phosphorylation of MDM2 to promote 53 ubiquitylation, which induces drug resistance, and increases eNOS to induce angiogenesis. The phosphorylation of ERK is stimulated by $\mathrm{NgBR}$ through the recruitment of Ras to the plasma membrane. Altogether, these potential mechanisms all induce carcinogenesis. However, $\mathrm{NgBR}$ may also mediate the phosphorylation of AMPK $\alpha$ and suppress the activation of LXR $\alpha$ to reduce the level of lipids, which have anticancer effects. Furthermore, NgBR may maintain normal N-glycosylation and inhibit ER stress and the UPR to induce a significant anticancer effect. NgBR, neurite outgrowth inhibitor B receptor receptor; MET, mesenchymal-to-epithelial transition; EMT, epithelial-to-mesenchymal transition; TGF- $\beta$, tumor growth factor $\beta$; ZEB1, zinc finger E-box binding homeobox 1; TWIST, twist family bHLH transcription factor 1; Akt, protein kinase B; phos, phosphorylated; MDM2, mouse double minute 2 homolog; mTOR, mechanistic target of rapamycin; eNOS, endothelial nitric oxide synthase; ERK, extracellular signal-regulated kinase; ACC-1, acetyl-coenzyme A carboxylase-1; AMPK $\alpha$, adenosine monophosphate-activated protein kinase $\alpha$; LXR $\alpha$, Liver X receptor $\alpha$; SCD-1, stearoyl coenzyme A desaturase-1; ABCA1, ATP-binding cassette subfamily A member 1; FASN, fatty acid synthase; SREBP-1c, sterol-regulatory element-binding protein; ER, estrogen receptor; UPR, unfolded protein response. 
reduce cell apoptosis and the effects of antioestrogen therapy by a mechanism similar to the UPR. Activation of the UPR is induced by tumour hypoxia and endoplasmic reticulum stress during breast cancer. Subsequently, the upregulated mRNA of $\mathrm{X}$-box binding protein may bind to oestrogen receptors and induce agonistic activities, and these agonistic activities are able to promote the transcription of the mRNA encoding the B-cell lymphoma 2 protein, which serves a crucial role in anti-apoptosis and the effects of antioestrogen therapy (77). A previous study on MM suggested that the most important oncogenic factor is a B-Raf proto-oncogene, serine/threonine kinase (BRAF) gene mutation. This mutation upregulates the Ras-Raf-MEK signalling pathway, which may produce carcinogenic effects on the human body (78). A recent study indicated that BRAF mutations also induce the consistent and modest activation of the UPR with GRP78 upregulation, which is considered a tumour marker of MM metastasis and recurrence. This mechanism may be considered an essential factor in MM metastasis (79).

\section{Conclusions and future perspectives}

The present review summarizes the expression, characteristics and biological functions of $\mathrm{NgBR}$ in numerous cancer types, which are complicated and novel and had remained unclear until now. This information is presented in the schematic representation presented in Fig. 1. Numerous pathophysiologic studies have confirmed that NgBR serves an important role in carcinogenesis and that its activation promotes cell proliferation and migration and inhibits apoptosis via multiple physiopathological mechanisms, including the AMPK $\alpha(11,69), \operatorname{LXR} \alpha(11,69)$, Akt $(20,43)$ and ERK $(28,43)$ signalling pathways.

$\mathrm{NgBR}$ offers novel clinical opportunities, but it is necessary for researchers to obtain an improved understanding of the mechanisms underlying the protective biological characteristics achieved following the elimination of drug resistance, which will improve the design of systemic therapies for numerous cancer types. To better understand the chemoresistance mediated by $\mathrm{NgBR}$, the characteristics of $\mathrm{NgBR}$ must be confirmed, including its size, shape, pathophysiological effect and structure-function associations $(15,43)$.

Novel studies demonstrated that $\mathrm{NgBR}$ activation serves a key role in reprogramming the metabolism of drug-resistant cancer cells, including promoting p53 ubiquitylation (15) and the UPR (12). In addition, $\mathrm{NgBR}$ has been demonstrated to be effective in facilitating the EMT, which has been confirmed to be a root factor affecting cancer chemosensitivity (18). $\mathrm{NgBR}$ inhibition may be a more powerful and promising tool for reversing chemoresistance compared with $\mathrm{NgBR}$ activation.

Furthermore, the development of highly selective NgBR inhibitors, including miR-26a, will not only promote further study on the molecular function of $\mathrm{NgBR}$ but also provide a potential approach for cancer therapy (53). Interestingly, doxycycline is able to induce the expression of inducible short hairpin RNA interference targeting $\mathrm{NgBR}$, which is currently being considered for use in cancer prevention and treatment (29). Doxycycline has been demonstrated to result in cell transformation and tumour growth arrest in breast cancer (80).

In contrast, certain molecular mechanisms underlying the anticancer properties of $\mathrm{NgBR}$ may be classified as part of the $\operatorname{LXR} \alpha, A M P K \alpha$ and N-glycosylation/endoplasmic reticulum stress/UPR pathways, which may aid maintenance of a normal cellular phenotype. The study of different tumour types and their treatments indicate that $\mathrm{NgBR}$ may be a tumour suppressor protein. Altogether, these results suggest that $\mathrm{NgBR}$ may serve a dual role in carcinogenesis. In the early stages, NgBR functions as an anticancer molecule, and its dysregulation may result in the initiation and development of cancer, whereas in the later stages, the activation of $\mathrm{NgBR}$ may help cancer cells escape from death and develop drug resistance $(11,12)$.

Therefore, ongoing and future studies are expected to elaborate on the fundamental structure-function associations of $\mathrm{NgBR}$ that may be useful for the development of novel drugs that directly target $\mathrm{NgBR}$ by providing available projects for the laboratory validation of its molecular function. Therefore, the genesis of contraindications postulates that $\mathrm{NgBR}$ may induce the rejuvenation of suppressed vascularity and heighten an opposite effect conducive to tumour growth. Furthermore, further clinical research of $\mathrm{NgBR}$ is also required to increase its stability in plasma, the transfer of one of its components to dehydrodolichyl diphosphate synthase subunit, the dynamics of its metabolism, its disease specificity, its cell type-specific targeting in cancer development and responses to conventional therapy.

\section{Acknowledgements}

Not applicable.

\section{Funding}

The present study was supported by the Hunan Provincial Natural Sciences Foundation of China (grant nos. 2017JJ2233 and 2018JJ2344), the Key Lab for Clinical Anatomy and Reproductive Medicine of Hengyang City (grant no. 2017KJ182) and the Science and Technology Plan Key Projects of Hunan Province (grant no. 2017SK2183).

\section{Availability of data and materials}

Not applicable.

\section{Author contributions}

YKL conceptualized the study, performed the analysis, collected data and participated in writing the paper. YJX collected data, acquired funding and participated in writing the paper. DCW participated in the collection of data and in the writing the paper. SLL performed the analysis and editing and also participated in the data collection. ST and ZCM reviewed and edited the paper and jointly designed the structure of the paper. All authors read and approved the final manuscript.

\section{Ethics approval and consent to participate}

Not applicable.

\section{Patient consent for publication}

Not applicable. 


\section{Competing interests}

The authors declare that they have no competing interests.

\section{References}

1. Miao RQ, Gao Y, Harrison KD, Prendergast J, Acevedo LM, Yu J, $\mathrm{Hu}$ F, Strittmatter SM and Sessa WC: Identification of a receptor necessary for Nogo-B stimulated chemotaxis and morphogenesis of endothelial cells. Proc Natl Acad Sci USA 103: 10997-11002, 2006.

2. Rana U, Liu Z, Kumar SN, Zhao B, Hu W, Bordas M, Cossette S, Szabo S, Foeckler J, Weiler H, et al: Nogo-B receptor deficiency causes cerebral vasculature defects during embryonic development in mice. Dev Biol 410: 190-201, 2016.

3. Long SL, Li YK, Xie YJ, Long ZF, Shi JF and Mo ZC: Neurite outgrowth inhibitor B receptor: A versatile receptor with multiple functions and actions. DNA Cell Biol 36: 1142-1150, 2017.

4. Dodd DA, Niederoest B, Bloechlinger S, Dupuis L, Loeffler JP and Schwab ME: Nogo-A, $-\mathrm{B}$, and $-\mathrm{C}$ are found on the cell surface and interact together in many different cell types. J Biol Chem 280: 12494-12502, 2005.

5. Li M and Song J: Nogo-B receptor possesses an intrinsically unstructured ectodomain and a partially folded cytoplasmic domain. Biochem Biophys Res Commun 360: 128-134, 2007.

6. Schwab ME: Nogo and axon regeneration. Curr Opin Neurobiol 14: 118-124, 2004

7. Holcomb J, Doughan M, Spellmon N, Lewis B, Perry E, Zhang Y, Nico L, Wan J, Chakravarthy S, Shang W, et al: SAXS analysis of a soluble cytosolic NgBR construct including extracellular and transmembrane domains. PLoS One 13: e0191371, 2018.

8. Szafranski P, Von Allmen GK, Graham BH, Wilfong AA, Kang SH, Ferreira JA, Upton SJ, Moeschler JB, Bi W, Rosenfeld JA, et al: 6q22.1 microdeletion and susceptibility to pediatric epilepsy. Eur J Hum Genet 23: 173-179, 2015.

9. Grabińska KA, Park EJ and Sessa WC: cis-prenyltransferase: new insights into protein glycosylation, rubber synthesis, and human diseases. J Biol Chem 291: 18582-18590, 2016.

10. Park EJ, Grabińska KA, Guan Z and Sessa WC: NgBR is essential for endothelial cell glycosylation and vascular development. EMBO Rep 17: 167-177, 2016.

11. Hu W, Zhang W, Chen Y, Rana U, Teng RJ, Duan Y, Liu Z, Zhao B, Foeckler J, Weiler H, et al: Nogo-B receptor deficiency increases liver $\mathrm{X}$ receptor alpha nuclear translocation and hepatic lipogenesis through an adenosine monophosphate-activated protein kinase alpha-dependent pathway. Hepatology 64: 1559-1576, 2016.

12. Park EJ, Grabińska KA, Guan Z, Stránecký V, Hartmannová H, Hodaňová K, Barešová $\mathrm{V}$, Sovová J, Jozsef L, Ondrušková N, et al: Mutation of Nogo-B receptor, a subunit of cis-prenyltransferase, causes a congenital disorder of glycosylation. Cell Metab 20: 448-457, 2014.

13. Eckharter C, Junker N, Winter L, Fischer I, Fogli B, Kistner S, Pfaller K, Zheng B, Wiche G, Klimaschewski L, et al: Schwann cell expressed Nogo-B modulates axonal branching of adult sensory neurons through the Nogo-B receptor NgBR. Front Cell Neurosci 9: 454, 2015.

14. Wang B, Zhao B, North P, Kong A, Huang J and Miao QR: Expression of $\mathrm{NgBR}$ is highly associated with estrogen receptor alpha and survivin in breast cancer. PLoS One 8: e78083, 2013.

15. Dong C, Zhao B, Long F, Liu Y, Liu Z, Li S, Yang X, Sun D, Wang H, Liu Q, et al: Nogo-B receptor promotes the chemoresistance of human hepatocellular carcinoma via the ubiquitination of p53 protein. Oncotarget 7: 8850-8865, 2016.

16. Calik J, Pula B, Piotrowska A, Wojnar A, Witkiewicz W, Grzegrzolka J, Podhorska-Okolow $\mathrm{M}$ and Dziegiel P: Prognostic significance of NOGO-A/B and NOGO-B receptor expression in malignant melanoma - A preliminary study. Anticancer Res 36: 3401-3407, 2016

17. Pula B, Werynska B, Olbromski M, Muszczynska-Bernhard B, Chabowski M, Janczak D, Zabel M, Podhorska-Okolow M and Dziegiel P: Expression of Nogo isoforms and Nogo-B receptor (NgBR) in non-small cell lung carcinomas. Anticancer Res 34: 4059-4068, 2014.

18. Zhao B, Xu B, Hu W, Song C, Wang F, Liu Z, Ye M, Zou H and Miao QR: Comprehensive proteome quantification reveals $\mathrm{NgBR}$ as a new regulator for epithelial-mesenchymal transition of breast tumor cells. J Proteomics 112: 38-52, 2015.
19. Siegel R, Naishadham D and Jemal A: Cancer statistics, 2013. CA Cancer J Clin 63: 11-30, 2013.

20. Pula B, Olbromski M, Owczarek T, Ambicka A, Witkiewicz W, Ugorski M, Rys J, Zabel M, Dziegiel P and PodhorskaOkolow M: Nogo-B receptor expression correlates negatively with malignancy grade and ki-67 antigen expression in invasive ductal breast carcinoma. Anticancer Res 34: 4819-4828, 2014.

21. Maluccio $M$ and Covey A: Recent progress in understanding, diagnosing, and treating hepatocellular carcinoma. CA Cancer $\mathbf{J}$ Clin 62: 394-399, 2012.

22. Kuo TC, Chang PY, Huang SF, Chou CK and Chao CC: Knockdown of HURP inhibits the proliferation of hepacellular carcinoma cells via downregulation of gankyrin and accumulation of p53. Biochem Pharmacol 83: 758-768, 2012.

23. Elder DE: Melanoma screening and mortality. J Natl Cancer Inst: Mar 29, 2018 (Epub ahead of print). doi: 10.1093/jnci/djy056. 2018

24. Lee JH, Miele ME, Hicks DJ, Phillips KK, Trent JM, Weissman BE and Welch DR: KiSS-1, a novel human malignant melanoma metastasis-suppressor gene. J Natl Cancer Inst 88: 1731-1737, 1996.

25. Wu D, Zhao B, Qi X, Peng F, Fu H, Chi X, Miao QR and Shao S: Nogo-B receptor promotes epithelial-mesenchymal transition in non-small cell lung cancer cells through the Ras/ERK/Snaill pathway. Cancer Lett 418: 135-146, 2018.

26. Cui J, He W, Yi B, Zhao H, Lu K, Ruan H and Ma D: mTOR pathway is involved in ADP-evoked astrocyte activation and ATP release in the spinal dorsal horn in a rat neuropathic pain model. Neuroscience 275: 395-403, 2014.

27. Zhao B, Chun C, Liu Z, Horswill MA, Pramanik K, Wilkinson GA, Ramchandran R and Miao RQ: Nogo-B receptor is essential for angiogenesis in zebrafish via Akt pathway. Blood 116: 5423-5433, 2010.

28. Zhao B, Hu W, Kumar S, Gonyo P, Rana U, Liu Z, Wang B, Duong WQ, Yang Z, Williams CL, et al: The Nogo-B receptor promotes Ras plasma membrane localization and activation. Oncogene 36: 3406-3416, 2017.

29. Inoue $K$ and Fry EA: Aberrant splicing of the DMP1-ARFMDM2-p53 pathway in cancer. Int J Cancer 139: 33-41, 2016.

30. Ryu HW, Shin DH, Lee DH, Won HR and Kwon SH: A potent hydroxamic acid-based, small-molecule inhibitor A452 preferentially inhibits HDAC6 activity and induces cytotoxicity toward cancer cells irrespective of p53 status. Carcinogenesis 39: 72-83, 2018.

31. Abraham AG and O'Neill E: PI3K/Akt-mediated regulation of p53 in cancer. Biochem Soc Trans 42: 798-803, 2014.

32. Jo M, Lester RD, Montel V, Eastman B, Takimoto S and Gonias SL: Reversibility of epithelial-mesenchymal transition (EMT) induced in breast cancer cells by activation of urokinase receptor-dependent cell signaling. J Biol Chem 284: 22825-22833, 2009.

33. Brabletz T: EMT and MET in metastasis: Where are the cancer stem cells? Cancer Cell 22: 699-701, 2012.

34. Larue L and Bellacosa A: Epithelial-mesenchymal transition in development and cancer: Role of phosphatidylinositol 3' kinase/ AKT pathways. Oncogene 24: 7443-7454, 2005.

35. Bakin AV, Tomlinson AK, Bhowmick NA, Moses HL and Arteaga CL: Phosphatidylinositol 3-kinase function is required for transforming growth factor beta-mediated epithelial to mesenchymal transition and cell migration. J Biol Chem 275: 36803-36810, 2000.

36. Wendt MK, Smith JA and Schiemann WP: Transforming growth factor- $\beta$-induced epithelial-mesenchymal transition facilitates epidermal growth factor-dependent breast cancer progression. Oncogene 29: 6485-6498, 2010.

37. Yang L, Han S and Sun Y: An IL6-STAT3 loop mediates resistance to PI3K inhibitors by inducing epithelial-mesenchymal transition and cancer stem cell expansion in human breast cancer cells. Biochem Biophys Res Commun 453: 582-587, 2014.

38. Song Q, Jiang S, Zhang X, Pan C, Lu C, Peng J and Li Q: Radiosensitivity of human ovarian cancer cells is enhanced by pseudolaric acid B due to the inhibition of the Ras/Raf/ERK signaling pathway. Exp Ther Med 15: 685-690, 2018.

39. Sriskanthadevan-Pirahas S, Lee J and Grewal SS: The EGF/ Ras pathway controls growth in Drosophila via ribosomal RNA synthesis. Dev Biol 439: 19-29, 2018.

40. Takahashi-Niki K, Kato-Ose I, Murata H, Maita H, Iguchi-Ariga SM and Ariga H: Epidermal growth factordependent activation of the extracellular signal-regulated kinase pathway by DJ-1 protein through its direct binding to c-Raf protein. J Biol Chem 290: 17838-17847, 2015. 
41. Rotblat B, Belanis L, Liang H, Haklai R, Elad-Zefadia G, Hancock JF, Kloog Y and Plowman SJ: H-Ras nanocluster stability regulates the magnitude of MAPK signal output. PLoS One 5: e11991, 2010

42. Roy S, Luetterforst R, Harding A, Apolloni A, Etheridge M, Stang E, Rolls B, Hancock JF and Parton RG: Dominant-negative caveolin inhibits H-Ras function by disrupting cholesterol-rich plasma membrane domains. Nat Cell Biol 1: 98-105, 1999.

43. Jin Y, Hu W, Liu T, Rana U, Aguilera-Barrantes I, Kong A, Kumar SN, Wang B, Gao P, Wang X, et al: Nogo-B receptor increases the resistance of estrogen receptor positive breast cancer to paclitaxel. Cancer Lett 419: 233-244, 2018.

44. Buday L and Downward J: Many faces of Ras activation. Biochim Biophys Acta 1786: 178-187, 2008

45. Prior IA and Hancock JF: Ras trafficking, localization and compartmentalized signalling. Semin Cell Dev Biol 23: 145-153, 2012.

46. Karadedou CT, Gomes AR, Chen J, Petkovic M, Ho KK, Zwolinska AK, Feltes A, Wong SY, Chan KY, Cheung YN, et al: FOXO3a represses VEGF expression through FOXM1-dependent and -independent mechanisms in breast cancer. Oncogene 31 : 1845-1858, 2012

47. Sherbet GV: Suppression of angiogenesis and tumour progression by combretastatin and derivatives. Cancer Lett 403: 289-295, 2017.

48. Mantovani G, Macciò A, Madeddu C, Gramignano G, Lusso MR, Serpe R, Massa E, Astara G and Deiana L: A phase II study with antioxidants, both in the diet and supplemented, pharmaconutritional support, progestagen, and anti-cyclooxygenase-2 showing efficacy and safety in patients with cancer-related anorexia/ cachexia and oxidative stress. Cancer Epidemiol Biomarkers Prev 15: 1030-1034, 2006.

49. Saharinen P, Eklund L, Pulkki K, Bono P and Alitalo K: VEGF and angiopoietin signaling in tumor angiogenesis and metastasis. Trends Mol Med 17: 347-362, 2011.

50. Vasudevan D and Thomas DD: Insights into the diverse effects of nitric oxide on tumor biology. Vitam Horm 96: 265-298, 2014

51. Ricciuti B, Foglietta J, Bianconi V, Sahebkar A and Pirro M: Enzymes involved in tumor-driven angiogenesis: A valuable target for anticancer therapy. Semin Cancer Biol: Nov 8. 2017 (Epub ahead of print). S1044-579X(17)30043-3, 2017. doi: 10.1016/j.semcancer.2017.11.005.

52. Teng RJ, Rana U, Afolayan AJ, Zhao B, Miao QR and Konduri GG: Nogo-B receptor modulates angiogenesis response of pulmonary artery endothelial cells through eNOS coupling. Am J Respir Cell Mol Biol 51: 169-177, 2014

53. Jo HN, Kang H, Lee A, Choi J, Chang W, Lee MS and Kim J: Endothelial miR-26a regulates VEGF-Nogo-B receptor-mediated angiogenesis. BMB Rep 50: 384-389, 2017.

54. Cruz P, Torres C, Ramírez ME, Epuñán MJ, Valladares LE and Sierralta WD: Proliferation of human mammary cancer cells exposed to 27-hydroxycholesterol. Exp Ther Med 1: 531-536, 2010.

55. Furberg AS, Veierød MB, Wilsgaard T, Bernstein L and Thune I: Serum high-density lipoprotein cholesterol, metabolic profile and breast cancer risk. J Natl Cancer Inst 96: 1152-1160, 2004.

56. Alikhani N, Ferguson RD, Novosyadlyy R, Gallagher EJ, Scheinman EJ, Yakar S and LeRoith D: Mammary tumor growth and pulmonary metastasis are enhanced in a hyperlipidemic mouse model. Oncogene 32: 961-967, 2013.

57. Ooi K, Shiraki K, Sakurai Y, Morishita Y and Nobori T: Clinical significance of abnormal lipoprotein patterns in liver diseases. Int J Mol Med 15: 655-660, 2005.

58. Rice SJ, Liu X, Miller B, Joshi M, Zhu J, Caruso C, Gilbert C, Toth J, Reed M, Rassaei N, et al: Proteomic profiling of human plasma identifies apolipoprotein E as being associated with smoking and a marker for squamous metaplasia of the lung. Proteomics 15: 3267-3277, 2015.

59. Repa JJ and Mangelsdorf DJ: The role of orphan nuclear receptors in the regulation of cholesterol homeostasis. Annu Rev Cell Dev Biol 16: 459-481, 2000

60. Shackelford DB: Unravelling the connection between metabolism and tumorigenesis through studies of the liver kinase B1 tumour suppressor. J Carcinog 12: 16, 2013.

61. Hardie DG, Schaffer BE and Brunet A: AMPK: An EnergySensing Pathway with Multiple Inputs and Outputs. Trends Cell Biol 26: 190-201, 2016.
62. Hardie DG: AMPK: A target for drugs and natural products with effects on both diabetes and cancer. Diabetes 62: 2164-2172, 2013.

63. He G, Zhang YW, Lee JH, Zeng SX, Wang YV, Luo Z, Dong XC, Viollet B, Wahl GM and Lu H: AMP-activated protein kinase induces p53 by phosphorylating MDMX and inhibiting its activity. Mol Cell Biol 34: 148-157, 2014.

64. Li W, Saud SM, Young MR, Chen G and Hua B: Targeting AMPK for cancer prevention and treatment. Oncotarget 6: 7365-7378, 2015.

65. Jones RG, Plas DR, Kubek S, Buzzai M, Mu J, Xu Y, Birnbaum MJ and Thompson CB: AMP-activated protein kinase induces a p53-dependent metabolic checkpoint. Mol Cell 18: 283-293, 2005.

66. Vander Heiden MG, Cantley LC and Thompson CB: Understanding the Warburg effect: The metabolic requirements of cell proliferation. Science 324: 1029-1033, 2009.

67. Handa N, Takagi T, Saijo S, Kishishita S, Takaya D, Toyama M, Terada T, Shirouzu M, Suzuki A, Lee S, et al: Structural basis for compound $\mathrm{C}$ inhibition of the human AMP-activated protein kinase $\alpha 2$ subunit kinase domain. Acta Crystallogr D Biol Crystallogr 67: 480-487, 2011.

68. Hadad SM, Baker L, Quinlan PR, Robertson KE, Bray SE, Thomson G, Kellock D, Jordan LB, Purdie CA, Hardie DG, et al: Histological evaluation of AMPK signalling in primary breast cancer. BMC Cancer 9: 307, 2009.

69. Zhang W, Yang X, Chen Y, Hu W, Liu L, Zhang X, Liu M, Sun L, Liu Y, Yu M, et al: Activation of hepatic Nogo-B receptor expression-A new anti-liver steatosis mechanism of statins. Biochim Biophys Acta 1863: 177-190, 2018.

70. Grabińska KA, Edani BH, Park EJ, Kraehling JR and Sessa WC: A conserved carboxy-terminal RxG motif in the NgBR subunit of cis-prenyltransferase is critical for prenyltransferase activity. J Biol Chem 292: 17351-17361, 2017.

71. Boersema PJ, Geiger T, Wisniewski JR and Mann M: Quantification of the N-glycosylated secretome by super-SILAC during breast cancer progression and in human blood samples. Mol Cell Proteomics 12: 158-171, 2013.

72. Li N, Xu H, Fan K, Liu X, Qi J, Zhao C, Yin P, Wang L, Li $\mathrm{Z}$ and Zha $\mathrm{X}$ : Altered $\beta 1,6-$ GlcNAc branched N-glycans impair TGF- $\beta$-mediated epithelial-to-mesenchymal transition through Smad signalling pathway in human lung cancer. J Cell Mol Med 18: 1975-1991, 2014

73. Chen CY, Jan YH, Juan YH, Yang CJ, Huang MS, Yu CJ, Yang PC, Hsiao M, Hsu TL and Wong CH: Fucosyltransferase 8 as a functional regulator of nonsmall cell lung cancer. Proc Natl Acad Sci USA 110: 630-635, 2013.

74. Uramoto H, Sugio K, Oyama T, Nakata S, Ono K, Yoshimastu T, Morita M and Yasumoto K: Expression of endoplasmic reticulum molecular chaperone Grp78 in human lung cancer and its clinical significance. Lung Cancer 49: 55-62, 2005.

75. Kim KM, Yu TK, Chu HH, Park HS, Jang KY, Moon WS, Kang MJ, Lee DG, Kim MH, Lee JH, et al: Expression of ER stress and autophagy-related molecules in human non-small cell lung cancer and premalignant lesions. Int J Cancer 131: E362-E370, 2012.

76. Lin Y, Wang Z, Liu L and Chen L: Akt is the downstream target of GRP78 in mediating cisplatin resistance in ER stress-tolerant human lung cancer cells. Lung Cancer 71: 291-297, 2011.

77. Clarke R, Cook KL, Hu R, Facey CO, Tavassoly I, Schwartz JL, Baumann WT, Tyson JJ, Xuan J, Wang Y, et al: Endoplasmic reticulum stress, the unfolded protein response, autophagy, and the integrated regulation of breast cancer cell fate. Cancer Res 72: 1321-1331, 2012

78. Meng XX, Yao M, Zhang XD, Xu HX and Dong Q: ER stressinduced autophagy in melanoma. Clin Exp Pharmacol Physiol 42: 811-816, 2015.

79. Martin S, Hill DS, Paton JC, Paton AW, Birch-Machin MA, Lovat PE and Redfern CP: Targeting GRP78 to enhance melanoma cell death. Pigment Cell Melanoma Res 23: 675-682, 2010

80. Matsumoto T, Uchiumi T, Monji K, Yagi M, Setoyama D, Amamoto R, Matsushima Y, Shiota M, Eto M and Kang D: Doxycycline induces apoptosis via ER stress selectively to cells with a cancer stem cell-like properties: Importance of stem cell plasticity. Oncogenesis 6: 397, 2017. 